\title{
Exploring random access and handshaking techniques in underwater wireless acoustic networks
}

\author{
Zhong Zhou $^{1 *}$, Zheng Peng ${ }^{1}$, Peng Xie ${ }^{1}$, Jun-Hong Cui ${ }^{1}$ and Zaihan Jiang ${ }^{2}$
}

\begin{abstract}
In this article, we study the medium access control (MAC) problem in underwater wireless acoustic networks. We explore the random access and handshaking (i.e., RTS/CTS) techniques in both single-channel and multi-channel network scenarios. We model and analyze these two approaches, and conduct extensive simulations to study their performance in various network conditions. Based on our results, we observe that the performance of both approaches are affected by many factors such as data rate, propagation delay and packet size. Our results show that the RTS/CTS approach is more suitable for dense networks with high date rate, whereas the random access approach is preferred in sparse networks with low data rate. Our results also demonstrate that multi-channel techniques can potentially help us combat the long delay feature of underwater acoustic channels. However, uncoordinated random channel access cannot fully exploit the advantages of the multi-channel network settings and it performs even worse than the single-channel random access protocol. Only with careful design and coordination such as multi-channel access with RTS/CTS handshaking process, can multi-channel MAC protocols greatly improve the system performance. We believe that this study will provide useful guidelines for efficient MAC design in underwater wireless acoustic networks.
\end{abstract}

\section{Introduction}

Recently, there has been a rapidly growing interest in monitoring underwater environments for scientific exploration, commercial exploitation, and coastline protection. The ideal vehicle for this type of extensive applications is a distributed underwater system with networked wireless nodes, referred to as underwater wireless networks $[1,2]$. However, due to the unique characteristics of underwater acoustic channels (such as limited available bandwidth, long propagation delay and extensive time-varying multi-path effects) and the harsh underwater environment, building autonomous underwater acoustic networks encounters grand challenges at almost every level of the protocol stack.

Since the inception of the concept of underwater networks, a large amount of research study has been conducted in this interesting research area. Readers can refer [1-5] for challenges and states-of-art for the research in

\footnotetext{
*Correspondence: zhz05002@engr.uconn.edu

${ }^{1}$ Computer Science \& Engineering Department, University of Connecticut,

Storrs, CT, USA

Full list of author information is available at the end of the article
}

underwater networks. New routing and medium access control (MAC) protocols were proposed in [6-11] to accommodate the unique characteristics of underwater acoustic networks. The authors of [12] investigated the synchronization problem for long delay acoustic channels of UWSN. In [13], the authors addressed the energy issues in UWSN and proposed methods to estimate the battery lifetime and power cost of shallow water networks. Reliable data transfer which is aimed to provide reliable service to the upper-layer applications has also been investigated in [14-16].

MAC protocols decide how multiple nodes share the underlying acoustic channel, which is critical to the overall network performance. In this article, we investigate the MAC protocols for underwater acoustic networks. Due to the long propagation delay of acoustic channels (the speed of sound in water is about $1500 \mathrm{~m} / \mathrm{s}$, five orders of magnitude lower than that of radio in air), directly adapting the MAC protocols designed for terrestrial radio networks to underwater environments would be very inefficient. The new challenges for the MAC design in underwater acoustic networks have

\section{黑 Springer}

2013 Zhou et al: licensee Springer. This is an Open Access article distributed under the terms of the Creative Commons

Attribution License (http://creativecommons.org/licenses/by/2.0), which permits unrestricted use, distribution, and reproduction in any medium, provided the original work is properly cited. 
recently inspired a significant amount of research efforts [17-24].

In general, MAC protocols can be roughly divided into two main categories: (1) contention-free protocols that avoid collisions among transmission nodes, and (2) contention-based protocols where nodes compete for a shared channel, resulting in the probabilistic coordination. Contention-free protocols include timedivision multiple access (TDMA), frequency division multiple access (FDMA) and code division multiple access (CDMA), where users are separated in time, frequency, or code domains. These protocols have been widely used in modern cellular communication systems. Contentionbased protocols include random access (ALOHA, slotted ALOHA) $[25,26]$, and collision avoidance with handshaking (MACA, MACAW) [27,28], which is the basis of several widely-used standards including IEEE 802.11.

In this article, we investigate the two key techniques: random access and handshaking (employed by contention-based protocols) for long-delay underwater acoustic networks. It has been argued that contentionbased protocols that rely on handshaking, i.e., RTS (Request-To-Send)/CTS (Clear-To-Send), are not appropriate in underwater communications $[29,30]$. The common cited reason is that the RTS/CTS protocol involves a long end-to-end delay, thus decreasing the network performance. Based on a similar argument, in [31], Rodoplu et al. proposed a random access based MAC protocol for underwater acoustic sensor networks, focusing on low duty cycle applications with relatively sparse sensor deployment. To our best knowledge, however, there is no formal comparison of the random access and handshaking techniques in underwater acoustic networks.

In addition, recent research in terrestrial radio networks on multi-channel MAC protocols, which use multiple channels in parallel for data transmissions, show us that such a parallelism can greatly improve the network throughput, decrease channel access delay and lower energy consumption [32-35]. Although multi-channel techniques are available for underwater acoustic communication $^{\text {a }}$, it is still not clear whether multi-channel MAC protocols have any benefits in the new long-delay underwater acoustic network scenario.

The questions we strive to answer in this article are: (1) Is random access an absolute winner? (2) Can RTS/CTS based protocols yield better performance than random access in any network conditions? (3) Do multi-channel technologies provide any benefits in the long-delay underwater environment? And if they do, how can we achieve the benefits of multichannel settings. In the rest of this article, we will quantitatively study the two schemes: random access and RTS/CTS in both single-channel and multi-channel network scenarios.
The rest of this article is organized as follows. In Section 2, we describe our network model and assumptions. And then we model and analyze random access and RTS/CTS in single-channel network scenarios in Section 3. In Section 4, we model and analyze random access and RTS/CTS in multi-channel network scenarios. Simulation results are presented in Section 5. Finally, we review some related study in Section 6, followed by our conclusions in Section 7.

\section{Network models and assumptions}

1. As in $[34,35]$, we consider a one-hop fully connected network, in which there are $n$ Nodes, which can hear each other. The traffic at every node is an independent identical (iid) Poisson process with parameter $\lambda$.

2. The total available bandwidth in the network is $R$. Thus, in the single-channel case, the bandwidth of the channel will be $R$. While for the multi-channel case, the bandwidth of the control channel is set to be $R_{c}$. The bandwidth of a data channel and the guard band between channels are denoted as $R_{d}$ and $R_{s}$, separately. And we can get $R=R_{c}+m R_{d}+m R_{s}$.

3. The average length of a control packet (RTS/CTS) is set to be $L_{c}$ and the average length of a data packet is set to be $L_{d}$. Thus, in the single-channel case, the average transmission time for a control packet $T_{c}=\frac{L_{c}}{R}$, and the average transmission time for the data packet is $T_{d}=\frac{L_{d}}{R}$. For multi-channel case, $T_{c}=\frac{L_{c}}{R_{c}}$ and $T_{d}=\frac{L_{d}}{R_{d}}$,

4. The average energy consumption for transmitting a single control packet is denoted as $\epsilon_{c}$, and the average energy consumption for transmitting a data packet is represented as $\epsilon_{d}$. And here we ignore the energy consumption on data receiving and processing ${ }^{\mathrm{b}}$.

\section{Random access and RTS/CTS in single-channel network scenarios}

\subsection{Modeling single-channel random access}

In this approach, a sender simply starts sending data packets whenever it has data ready for delivery. When a data packet arrives at the receiver, if the receiver is not receiving any other packets and, during the time period of receiving this data packet, there is no incoming data packet (i.e., in a time period of $2 \times T_{d}$, there are no other arriving packets), then the receiver can receive this data packet successfully.

To compute the throughput and communication overhead of this approach, we first evaluate the success probability (denoted by $P_{s}$ ) of one packet sent from one sender (e.g., node $A$ ) to the receiver (node $B$ ). In fact, $P_{s}$ is equivalent to the probability that all the nodes (including node $A$ ) in the transmission range of node $B$ do not send any 
packets in a time period of $2 \times T_{d}$. If we denote the arriving time of the packet from $A$ as $t_{0}$, then for each sender $i$, the time period of $2 \times T_{d}$ is $\left[t_{0}-T_{d}-\tau_{i}, t_{0}+T_{d}-\tau_{i}\right]$, where $\tau_{i}$ is the propagation delay from node $i$ to node $B$. Please note that $\tau_{i}$ s are significant, as is different in radiobased networks. Considering the Poisson process of data generation at senders, the probability $P_{S}$ can be given by

$$
P_{s}=\Pi P_{i}=\Pi e^{-2 \times \lambda \times T_{d}}=e^{-2(n-1) \lambda T_{d}} .
$$

Then, the average throughput of the whole network measured in bits per second can be calculated as

$$
\eta=(n-1) \times \lambda \times L_{d} \times e^{-2(n-1) \lambda T_{d}} .
$$

Correspondingly, the energy consumption for every successful data packet will be evaluated as

$$
\epsilon=\frac{1}{P_{s}}=\epsilon_{d} e^{2(n-1) \lambda T_{d}} .
$$

where $\epsilon_{d}$ is the average energy consumption for transmitting a data packet.

\subsection{Modeling single-channel RTS/CTS}

The basic idea of the RTS/CTS scheme is that a sender has to capture the channel (by handshaking) before sending any data. In underwater acoustic networks, due to the long propagation delay, the traditional RTS/CTS model for radio-based networks should be modified. In the following, we use two examples to show why it is infeasible to directly use the traditional RTS/CTS model in underwater acoustic networks.

We first examine the example illustrated in Figure 1, where node $A$ and node $B$ are 60 meters apart and node $B$ and node $C$ are 90 meters apart. Assuming node $A$ sends an RTS to node $B$, and then node $B$ replies with a CTS. Considering the propagation speed of acoustic signal in water $(v=1500 \mathrm{~m} / \mathrm{s})$, it takes $\frac{60}{1500}=40 \mathrm{~ms}$ for the CTS to arrive at node $A$ and $\frac{90}{1500}=60 \mathrm{~ms}$ to arrive at node $C$. After node $A$ receives the CTS, node $A$ cannot send data immediately since node $C$ has not received the CTS from node $B$ yet and possibly sends an RTS to node $B$, which will collide with the data packets sent from node $A$.

Thus, in underwater acoustic networks, to make RTS/CTS work, as in [10], we make the following changes:

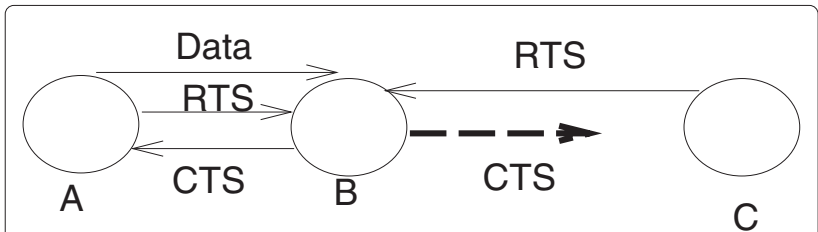

Figure 1 RTS/CTS Example 1. when a node receives a CTS, it cannot send data immediately. Instead, it has to wait for the CTS to propagate the whole transmission range of the receiver (i.e., the sender of the CTS).

In underwater acoustic networks, though the long propagation delay significantly damages the effectiveness of the traditional RTS/CTS mechanism, this network feature allows RTS/CTS exchange and data communication to proceed in parallel. As shown in Figure 2, while node $B$ is receiving a data packet from node $A$, node $C$ can schedule to send an RTS to node $B$. When this RTS propagates to node $B$, node $B$ just finishes receiving data from node $A$ and is now ready for the RTS from node $C$.

We now evaluate the throughput of our modified RTS/CTS approach. In this approach, the time for one transmission, $T_{\theta}$, is calculated as $\tau_{\text {cts }}+\tau+T_{d}$, where $\tau_{\text {cts }}$ is the time that the CTS propagates through the transmission range of the receiver, $\tau$ is the time that data propagates from the sender to the receiver (i.e., $\tau=d / v$ ) and $T_{d}$ is the data transmission time. Due to the effective collision avoidance of RTS/CTS, when the data rate is higher than the channel capacity, the effective data rate for the receiver reaches the limit, and cannot increase any more. Thus, the effective data rate, $\lambda_{t}$, for the receiver can be calculated as $\min \left(\frac{1}{T_{\theta}},(n-1) \times \lambda\right)$. Then the throughput of RTS/CTS is evaluated as

$$
\eta=L_{d} \times \lambda_{t}
$$

As for the energy consumption, it can be easily obtained as follows:

$$
\epsilon=2 \times \epsilon_{c}+\epsilon_{d}
$$

where $\epsilon_{c}$ is the average energy consumption for transmitting a control packet and $\epsilon_{d}$ is the energy consumption for transmitting a data packet. Please note that in the computation, we ignore the collision of RTS/CTS packets, since in the single-channel case, $T_{c}$ is usually quite small, the collision probability of RTS/CTS packets is very low ${ }^{\mathrm{c}}$.

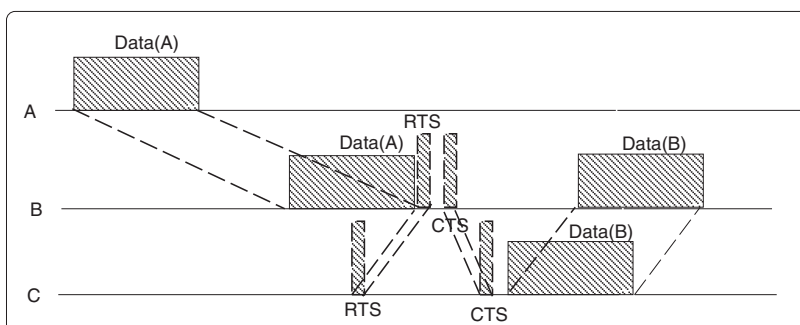

Figure 2 RTS/CTS Example 2. 


\section{Random access and RTS/CTS in multi-channel network scenarios}

\subsection{System model}

Different from single-channel MAC protocols, multichannel MAC protocols utilize more than one data channels for data transmission. In such a MAC protocol, nodes will exchange control messages in order to negotiate the channel for data transmission. Data packets will later be sent on the agreed channel. Without losing generality, in this article, we assume one common control channel is dedicated for control messages and every node continuously monitors this channel.

\subsubsection{Multi-channel random access}

This is the simplest multi-channel MAC scheme. As shown in Figure 3, channels are divided into one control channel and multiple data channels. When a node wants to send a data packet, it first randomly selects a channel from the available data channels. A control packet will then be sent on the control channel to inform the receiver of the selected channel for data transmissions. After that, the data packet will then be sent on the selected data channel without any delay. No handshaking processes are required. As we can see, the control channel essentially uses the single-channel random access approach in Section 3.

\subsubsection{Multi-channel RTS/CTS}

This protocol is a combination of multi-channel access and the RTS/CTS technique. As shown in Figure 4, there are one common control channel and multiple data channels. Similar to the multi-channel with Aloha protocol, every node continuously monitors the control channel. The difference is that the node with outgoing packets will send a request-to-send (RTS) message on the control channel. The RTS message should include the sender/receiver id, the available channel set and the packet length. After correctly receiving a RTS message, the intended receiver will select one channel randomly

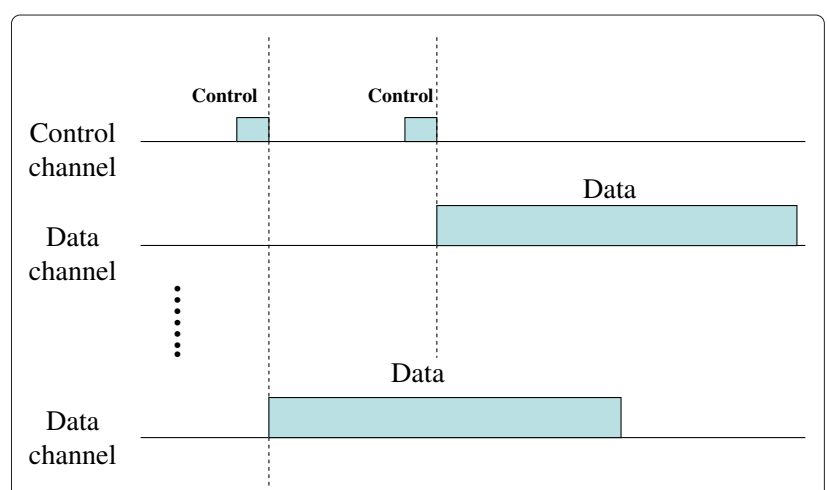

Figure 3 Multi-channel with aloha.

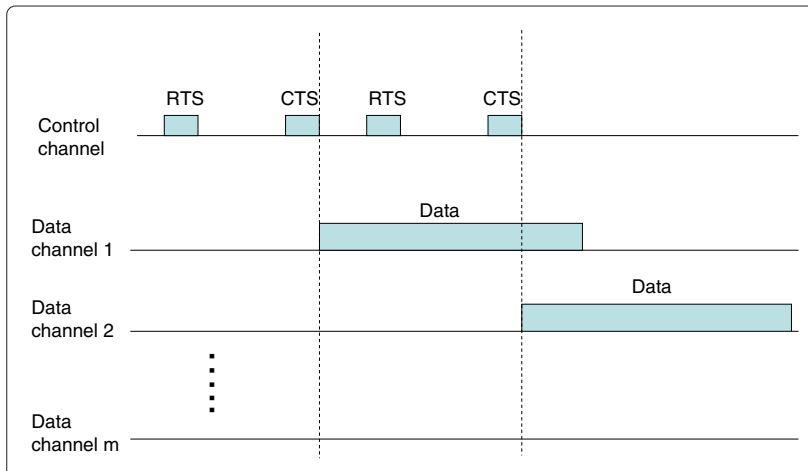

Figure 4 Multi-channel with RTS/CTS.

from the available channel set based on its network condition. Then, the receiver will respond with a clear-to-send (CTS) message to inform the sender of the selected data channel and tune to that channel. Upon receiving the CTS message, the sender will send out its data packet on the selected data channel. Through this RTS/CTS exchange process, overhearing neighbors will know this transmission event and the used data channel ${ }^{\mathrm{d}}$. This multi-channel with RTS/CTS protocol is similar to the multi-channel MAC protocol presented in [35], which is for terrestrial wireless networks and ignores the signal propagation delay. However. Due to the long propagation delay of underwater acoustic channels, the modeling and analysis process in this article is different from that in [35].

\subsection{Modeling multi-channel random access}

In this scheme, the probability of successfully transmitting one data packet can be denoted as

$$
P_{s}=P(A B)=P(A) P(B \mid A),
$$

where event $A$ means that there are no collisions for the control packet in the control channel and the control packet can be correctly received by its intended receiver (for simplicity, we assume that a packet will be successfully received by the receiver if there are no collisions for this packet), and event $B$ means that there are no collisions for the data packet on the selected data channel. The packet arriving process for a node is an independent identical Poisson process with parameter $\lambda$, then the total traffic in the network with $n$ nodes can be viewed as a Poisson process with parameter $n \lambda$.

If a control packet is transmitted at time $t_{c}$, in order for this control packet to be received correctly, there should be no other control packets in the network during $\left(t_{c}-\right.$ $T_{c}, t_{c}+T_{c}$ ), where $T_{c}$ is the duration of one control packet (as defined earlier). Thus we can have

$$
P(A)=e^{-2 n \lambda T_{c}} .
$$


For a data packet, even if its corresponding control packet has been successfully received, collisions can still happen. If another sender selects the same data channel within a very short time, the data packet will still get overlapped in the time domain and a collision occurs. Given a data packet, we use $x$ to denote the number of other data packets overlapping with it in the time domain (but their control packets do not collide with each other). Then we have

$$
P(x=k)=\frac{\left(2 n \lambda\left(T_{d}-T_{c}\right)\right)^{k}}{k !} e^{-2 n \lambda\left(T_{d}-T_{c}\right)} .
$$

Under the condition of $x=k$, the probability that none of these $k$ data packets selects the same channel as this packet is $\left(\frac{m-1}{m}\right)^{k}$ (since there are $m$ data channels in the network and every node selects its own data channel randomly and independently). Therefore, we can get

$$
\begin{aligned}
P(B \mid A) & =\sum_{k \in(0,1,2, \ldots)} P(x=k) \times\left(\frac{m-1}{m}\right)^{k} \\
& =\sum_{k \in(0,1,2, \ldots)}\left[\left(\frac{m-1}{m}\right)^{k}\right] \frac{\left(2 n \lambda\left(T_{d}-T_{c}\right)\right)^{k}}{k !} e^{-2 n \lambda\left(T_{d}-T_{c}\right)} .
\end{aligned}
$$

Now we are ready to derive the average successful transmission probability for one data packet $P_{S}$ :

$$
\begin{aligned}
P_{s}= & P(A B)= \\
=e^{-2 n \lambda T_{c}} & \times\left\{\sum_{k \in(0,1,2, \ldots)}\left[\left(\frac{m-1}{m}\right)^{k}\right]\right. \\
& \left.\times \frac{\left(2 n \lambda\left(T_{d}-T_{c}\right)\right)^{k}}{k !} e^{-2 n \lambda\left(T_{d}-T_{c}\right)}\right\} \\
=e^{-2 n \lambda T_{c}} & \times\left[e^{\left(\frac{-2 n \lambda\left(T_{d}-T_{c}\right)}{m}\right)}\right]=e^{-\frac{-2 n \lambda\left(T_{d}+(m-1) T_{c}\right)}{m}} .
\end{aligned}
$$

The average throughput $\eta$ can be derived as

$$
\eta=n \lambda L_{d} P_{s}=n \lambda L_{d} e^{\frac{-2 n \lambda\left(T_{d}+(m-1) T_{\mathcal{C}}\right)}{m}} .
$$

Equation (11) shows that the throughput of this protocol is not affected by the long-delay feature of underlying acoustic channels (since no propagation delay is involved in the throughput calculation).

In this multi-channel random access protocol, since every retransmission of a data packet is accompanied by the retransmission of the corresponding control packet, we can get $\alpha=\frac{1}{P_{s}}$. The average energy consumption per successful data packet thus can be derived as

$$
\epsilon=\frac{1}{P_{s}}\left(\epsilon_{c}+\epsilon_{d}\right)=e^{-\frac{-2 n \lambda\left(T_{d}+(m-1) T_{c}\right)}{m}} \times\left(\epsilon_{c}+\epsilon_{d}\right) .
$$

To obtain the optimal bandwidth allocation, we combine Equation (11) with Equation (12). By using Lagrange method [36], we can get

$$
R_{c}=\frac{R \sqrt{L_{c}}}{\sqrt{L_{d}}+\sqrt{L_{c}}}
$$

\subsection{Modeling multi-channel RTS/CTS}

For this protocol, we first analyze the control channel and obtain the effective input traffic to the data channels. Then, we analyze the data channels and propose two tractable Markov models [37] which can be served as the upper and lower bound systems.

\subsubsection{Analyzing control channel}

The control channel is basically a random channel with every successful RTS packet leading to a CTS packet transmission $^{\mathrm{e}}$. It has been shown that the completion time of successful RTS/CTS dialogues can be accurately modeled by a Poisson process $[35,38]$. Further, as shown in Section 3, the performance of a pure random protocol on a single control channel is not affected by long propagation delays. Hence, in the following, we use the same method as in [35] to model the control channel for multi-channel with RTS/CTS.

Since every data packet is preceded by a RTS message, the RTS message destined to a node is thus subject to the same Poisson process as the input traffic. Let us denote the average collision probability on the control channel as $P_{c}^{c}$. Then the CTS message on the control channel can be approximated as a Poisson process with parameter $\left(1-P_{c}^{c}\right) n \lambda$. Since collisions might happen between any RTS and CTS messages on the control channel, $P_{c}^{c}$ then can be derived as

$$
P_{c}^{c}=1-e^{-2 n\left(1+1-P_{c}^{c}\right) \lambda T_{c}} .
$$

Given $T_{c}$ and $\lambda$, we can obtain $P_{c}^{c}$ numerically. Since only successful RTS/CTS dialogues can result in data transmissions on the data channel, the effective input traffic in data channels can then be modeled as a Poisson process with parameter $\lambda_{e}$ :

$$
\lambda_{e}=\left(1-P_{c}^{c}\right)^{2} n \lambda
$$

\subsubsection{Analyzing data channels}

Because of long propagation delays, the RTS/CTS mechanism on the control channel can not eliminate the collision on the data channels. For example, as shown in Figure 5 , node $A$ has packets for node $B$ and node $C$ has packets for node $D$. Here, $C$ sends out a $R T S$ message to $D$ first. After $D$ receives the RTS message from $C$, it will choose one channel from the available channels and respond to $C$ with a CTS message. However, before the CTS message arrives at $C$, the RTS message of $A$ arrives 


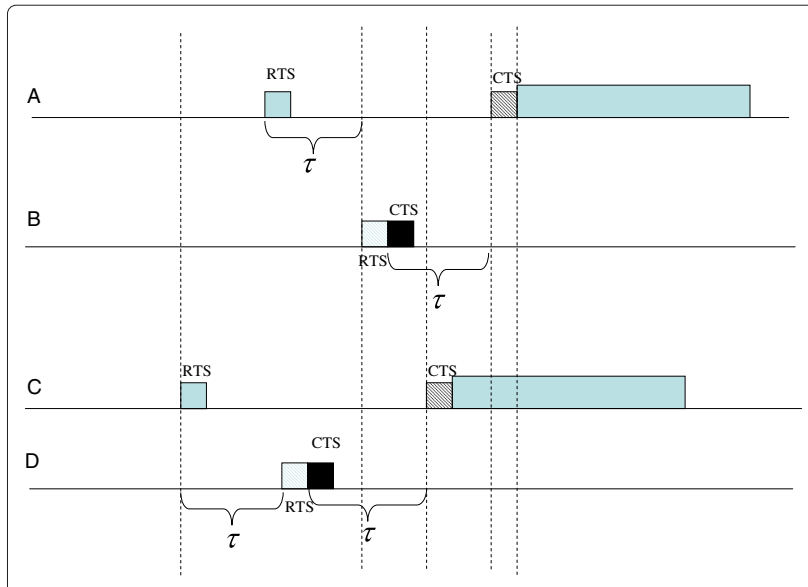

Figure 5 Node interaction in multi-channel with RTS/CTS.

at $B$ and $B$ will select one channel from its current available channel set. Since at this time, $B$ does not know which channel has been selected by $D$ to communicate with $C$, with certain probability, $B$ will select the same channel as $D$. In this case, collisions might happen on the selected data channel. For a data packet at time $t_{1}$, it may collide with a packet in $\left[t_{1}-\tau, t_{1}+\tau\right]$, where $\tau$ is the propagation delay. Thus, the collision window of one data packet in this protocol becomes $2 \tau$.

In terrestrial radio networks, the analysis process for the multi-channel RTS/CTS scheme directly models the data transmission process as a Markov chain $[35,38]$, which is a random discrete system that satisfies the Markov property [37]. Markov property states that the conditional probability distribution for the system at the next step (and in fact at all future steps) depends only on the current state of the system, and not additionally on the state of the system at previous steps [37]. In underwater acoustic networks, however, the long propagation delay of acoustic channels makes the data channel assignment process a quite complicated distributed stochastic process: the channel selection decision by a node based on its own perceived network condition may not be accurate. In fact, the data channel selection process for long delay networks can no longer be modeled by a Markov process since the future state of the network is not only related to its current state, but also related to the state $\tau$ before. To make the analysis of such a complicated system tractable, instead of modeling it directly, we try to find its upper and lower bounds.

\subsubsection{Lower bound and upper bound}

In this section, we first construct a "virtual" system so that its performance is equivalent to that of the original system. Then we develop lower and upper bounds for the virtual system.
Virtual system let us imagine a system with $m$ data channels, with each possessing the same bandwidth as the data channels in the original system. Time is slotted into a series of $2 \tau$ intervals, where $\tau$ is the propagation delay (Here we assume that the propagation delays between nodes are the same. Later, we will show that our analysis method can be easily extended to find the upper and lower bounds for networks with various propagation delays). The input traffic to this system is a Poisson process with parameter $\lambda_{e}=\left(1-P_{c}^{c}\right)^{2} n \lambda$, which is the same as the effective input traffic to the data channels of the original system. But for this virtual system, packet collisions are confined to one slot only, which means that every packet has no knowledge of other packets in its own slot and will possibly select the same channel as others do. However, every packet knows all packets outside of its own slot and thus collisions never happen for packets from different slots.

Figure 6 illustrates this virtual system. In the figure, packet 1 selects channel 1 for its transmission. Packet 2 , located in the same slot, does not know channel 1 being selected and may select channel 1 as well, which leads to a collision on channel 1 . For packet 3 , since it is in the next slot, it knows channels used by packet 1 and packet 2 and thus will not select the same channel. In the figure, packet 3 selects channel 2 for transmission, which is different from channel 1 selected by both packet 1 and packet 2 .

As shown in Figure 7, the only difference between this virtual system and the original system is that the collision region of the packet at any time $t_{1}$ is shifted from $\left[t_{1}-\tau, t_{1}+\tau\right]$ to $\left[\left\lfloor\frac{t_{1}}{2 \tau}\right\rfloor 2 \tau,\left\lfloor\frac{t_{1}}{2 \tau}+1\right\rfloor 2 \tau\right]$. Because the effective input traffic of both systems is the same timeinvariant Poisson process and the collision regions of both systems are of the same length $2 \tau$, the performance of this virtual system will be the same as that of the original system.

The virtual system is still too complicated to analyze directly: three continuous random processes interact in

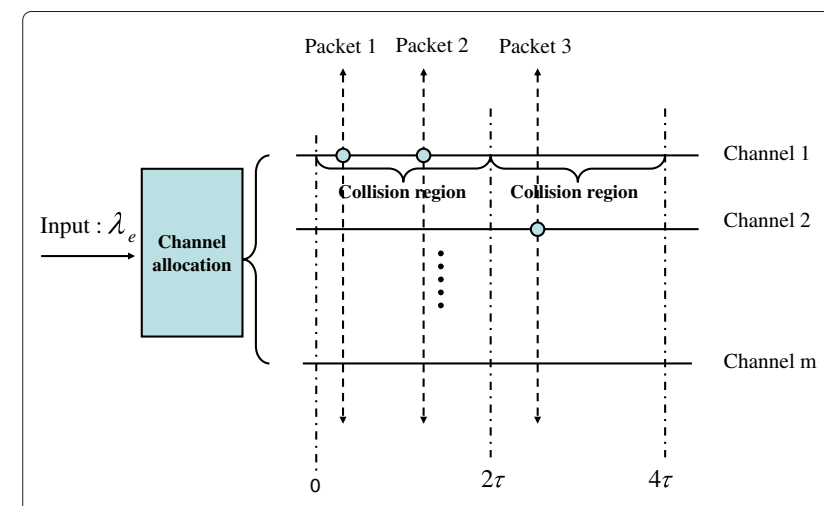

Figure 6 Illustration of virtual system. 


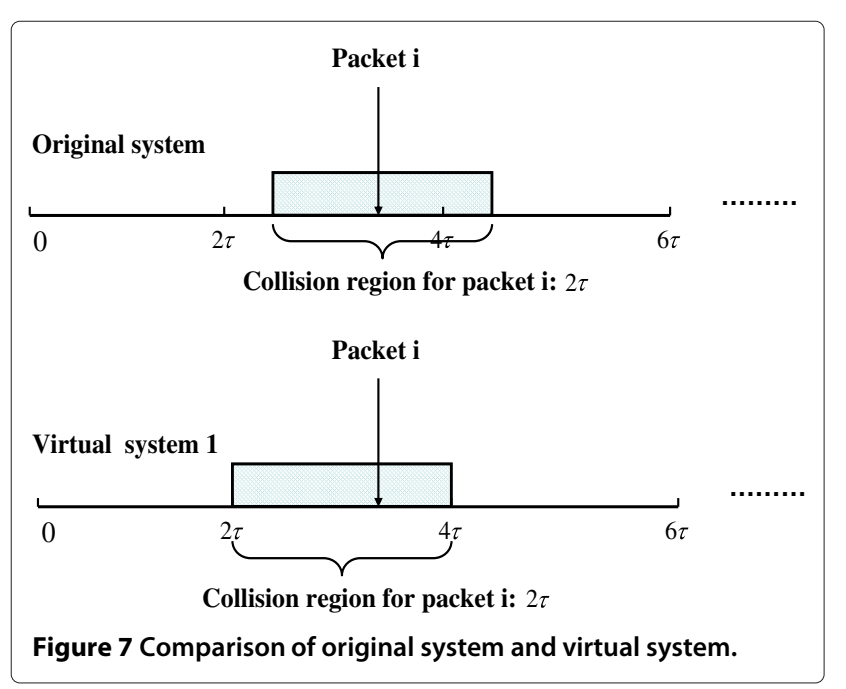

the system. The first is the packet arriving process which is a Poisson process; the second one is the packet leaving (channel recycle and reuse) process; and the third one is the channel allocation process in which one packet selects one channel randomly from its perceived available channel set. Fortunately, we could derive lower bound and upper bound for the virtual system. Correspondingly, we could obtain the lower bound and upper bound for the original system.

Lower bound system we confine the virtual system as follows: the available channel set of every packet will keep the same as that in the beginning of a slot. The channels that are released can be reused during a slot in the virtual system. However, this will not happen in this lower bound system. The released channels in this slot are only available to the packets in the next slot. Compared to the previous virtual system, the number of available channels for every packet in every slot is smaller because the released channels in this slot will not be available to the packets in the same slot any more. Thus, this confined system will have higher collision probability than the virtual system. It can be served as the lower bound of the virtual system.

Upper bound system we revise the virtual system as follows: the release of channels occurs at the beginning of a slot and thus these channels are available to all packets in this slot. Compared with the virtual system, the number of the available channels for every packet in one slot will be larger because all released channels will be available for all packets in the slot. Thus, this revised system must have lower collision probability and can be served as the upper bound of the virtual system.

Solving lower bound system for the lower bound system, we set the system state as the number of the available channels for the current slot. We use $P_{\text {st }}=$ $\left(p_{0}, p_{1}, \ldots, p_{i}, \ldots, p_{m}\right)$ to denote the system stationary probability, where $p_{i}$ means that there are $i$ channels available for the current slot. We have proved that this virtual lower bound system can be accurately modeled by discrete time Markov chains. We have also calculated its stationary probability vector $P_{\mathrm{st}}$ and the average data packet collision probability $p_{\mathrm{col}}$. The proofs and calculation procedures are in Appendix 1.

The overall packet success probability can thereby be denoted as

$$
P_{s}=1-p_{\text {col }}-p_{0},
$$

where $P_{0}$ is the average probability that there is no channel available for the system, the condition when the system can not support any data transmission.

The system average throughput can be calculated as

$$
\eta=\left(1-p_{\mathrm{col}}-p_{0}\right) n \lambda_{e} L_{d}
$$

Considering the RTS/CTS exchange process on the control channel as well as the collision behaviors on the data channels, we can get the average number of transmissions of control packets for one successfully transmitted data packet $\alpha$ as

$$
\alpha=\frac{2}{P_{s}\left(1-P_{c}^{c}\right)} .
$$

Accordingly, the average energy consumption per successful data packet for this system can be written as,

$$
\epsilon=\frac{2 \epsilon_{c}}{P_{s}\left(1-P_{c}^{c}\right)}+\frac{1}{P_{s}} \epsilon_{d} .
$$

The upper bound system can be solved in a similar way.

Handling various delays with the increase of the propagation delay, the system's collision region will increases and its performance will degrade. Thus, for a network with various propagation delays falling within $\left[\tau_{\min }, \tau_{\max }\right]$, we can use $\tau_{\max }$ as a parameter to get its lower bound and use $\tau_{\min }$ to get its upper bound. In Section 5, through simulations we will show that these bounds are quite tight in most cases.

\section{Simulation results}

In this section, we conduct simulations to compare the throughput and the energy consumptions of random access and RTS/CTS under various network conditions.

\subsection{Simulation settings}

In all simulations, unless specified otherwise, we set the bandwidth of the network $R$ to be $20 \mathrm{kbps}$. Thus, for the single-channel case, there is only one channel with bandwidth $20 \mathrm{kbps}$ available to every node. For the multichannel case, the bandwidth of the control channel is $3 \mathrm{kbps}$, which is chosen based on our simulation results in Section 5.1.1. The number of data channels is 16. And 
we set the band gap between channels to be $R_{s}=0.1 R_{d}$, Thus, the bandwidth for the data channel is about $1 \mathrm{kbps}$. The propagation speed of the acoustic signal is $1500 \mathrm{~m} / \mathrm{s}$ and the distance between any nodes follows a uniform distribution between 400 and $500 \mathrm{~m}$. Thus, the propagation delay between any nodes has a uniform distribution between $0.27 \mathrm{~s}$ and $0.33 \mathrm{~s}$. The average data packet and control packet length are 125 bytes and 10 bytes, respectively.

\subsubsection{Optimal bandwidth allocation for multi-channel with Aloha}

In this set of simulations, we vary the input traffic rate of every node to be $0.05,0.1$, and 0.2 packets per second respectively. The bandwidth of the control channel increases from $1 \mathrm{kbps}$ to $7 \mathrm{kbps}$. Figure 8 shows that an optimal bandwidth allocation between the control and the data channels does exist. It is $3 \mathrm{kbps}$ and does not change with the input traffic. And the theoretical results are very close to the simulation results, which indicates that our theoretical analysis can estimate the system performance very well.

\subsection{Does multi-channel work underwater?}

In this set of simulations, we compare multi-channel MAC protocols with their corresponding single-channel protocols. To make a fair comparison. We fix the input traffic rate of every node to be 0.1 packet per second and change the bandwidth of the control channel in multichannel MAC protocols.

Figure 9 shows that the average throughput of the multi-channel random protocol is less than that of the single-channel random protocol. And the single channel random protocols also shows big advantages on the energy efficiency. The inferiority of the multi-channel random

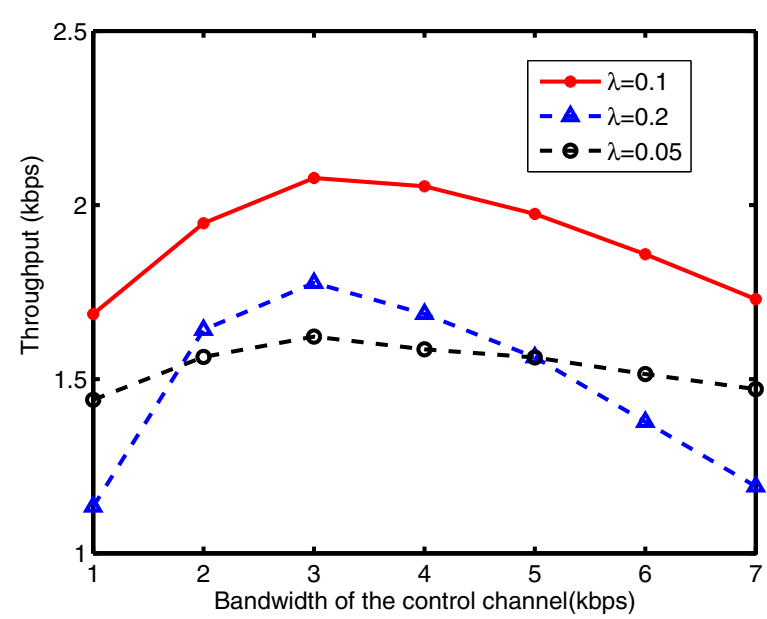

Figure 8 Throughput with varying control channel bandwidth for "Aloha". access protocol lies in its random access nature, its additional channel negotiation process on the control channel and the costs for the multi-channel settings such as the guard band among channels. This result clearly shows that without any effective coordinations among nodes, randomly accessing multiple channels cannot provide any benefits.

On the contrary, Figure 10 demonstrates that Multichannel RTS/CTS can achieve much higher throughput than the single-channel RTS/CTS protocol. For example, when the number of channels is 16 and the bandwidth of the control channel is $6 \mathrm{kbps}$, Multi-channel RTS/CTS can achieve throughput as high as $3.3 \mathrm{kbps}$, which is almost twice of that of the single channel RTS/CTS protocol. The advantages of Multi-channel with RTS/CTS lie in the parallelism of multiple channels. Since the handshaking process is conducted on a dedicated control channel and data packets are transmitted on data channels, the handshaking process can happen in parallel with the data transmission. In this way, the adverse effects of the long propagation delay of underwater acoustic channels can be effectively suppressed, which contributes to the high performance of this multi-channel MAC protocol. Figure 10 also shows us that the energy efficiency of the multichannel RTS/CTS is comparable to or even better than the single-channel RTS/CTS in some settings. From these results, it is evident that multi-channel settings could give us more freedom to combat the long delay underwater acoustic environment. In other words, with careful design, multi-channel MAC protocols can potentially achieve much better performance than the single-channel ones.

\subsubsection{Upper and lower bounds for multi-channel RTS/CTS}

In this set of simulations, we verify our theoretical upper and lower bounds. The upper and lower bounds are obtained by solving the Markov models in Section 4.3. And we also compare our results with the theoretical results of the ideal case which does not consider the propagation delay. These ideal theoretical results can be obtained by using the method from [35] and are referred to as "ideal case without delay" in the results.

We set the input traffic $\lambda$ to 0.2 packets per second and plot the results in Figure 11. From this figure, we can clearly see that our theoretical upper and lower bounds are quite tight. Further, compared with the theoretical results for the ideal case without delay, our theoretical bounds are much more accurate in long delay underwater acoustic networks.

Another trend we can observe from Figure 11 is that the throughput increases significantly with the lifting number of data channels and the average energy consumption decrease monotonically. Further, with the increase of the number of data channels, the system will approach the 

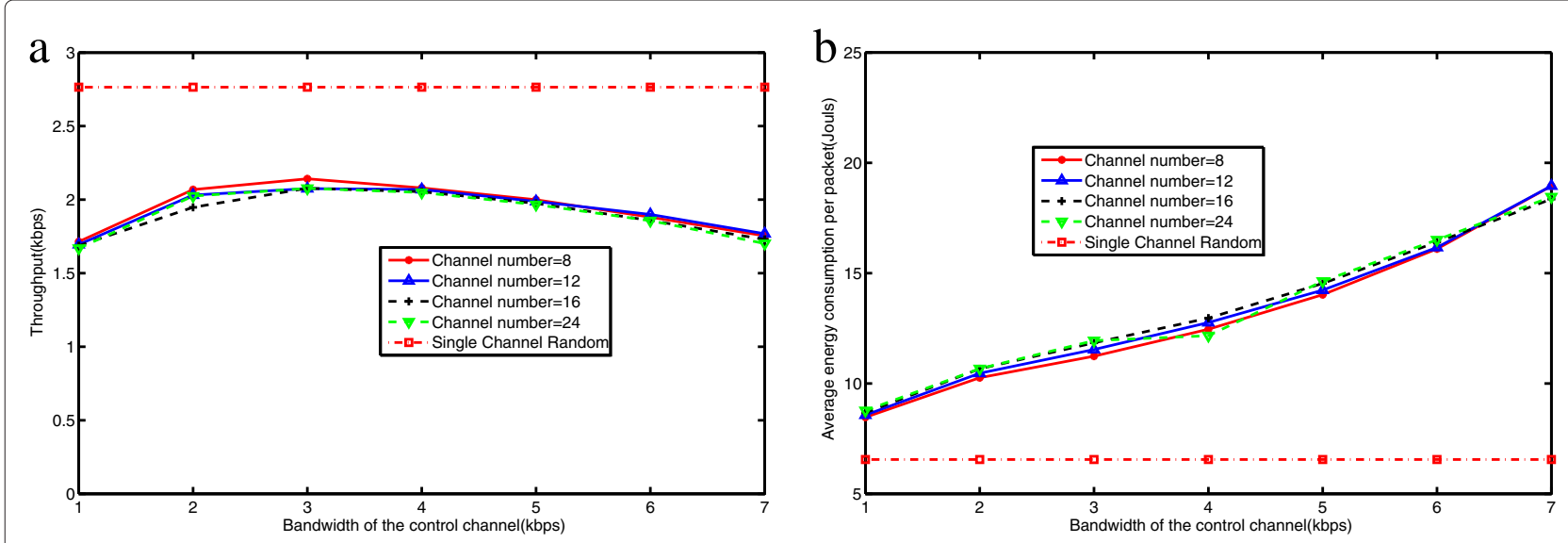

Figure 9 Comparison of random access for single-channel and multi-channel. (a) Comparison of throughput. (b) Comparison of energy consumption.

ideal case of zero propagation delay and achieve better throughput and energy efficiency. For example, when the number of data channels is 4 , the throughput gap between the actual system and the ideal system is $1.1 \mathrm{kbps}$. When the number of data channels increases to 32 , this gap is reduced to $0.2 \mathrm{kbps}$. This result suggests that the impact of long propagation delays can be alleviated by increasing the number of data channels.

\subsubsection{Effects of input traffic}

We change the input traffic of every node from 0.05 to 0.5 packet per second. The results are plotted in Figure 12. From this figure, we can observe that random access protocols in both single-channel and multi-channel networks demonstrate the same trend. The network throughput for these protocols will first increase with the input traffic. However, with the further increase of the input traffic, collisions among data packets will significantly degrade the system's performance, which makes the network throughput and the energy efficiency degrade sharply. While in the low traffic region, the collision probability introduced by the random access is small and will not affect the system performance much. As shown in Figure 12, random access can achieve comparable or even better performance than the RTS/CTS schemes which need an extra handshaking process when the input traffic is low.

For the RTS/CTS scheme, its throughput increase rapidly with the input traffic at first. And when the input traffic is high, it behaves much more stable than the random access schemes because of the RTS/CTS handshaking process which suppresses the collisions on the data packet. Figure 12 also shows us that compared with the random access schemes, RTS/CTS schemes can achieve much higher throughput and energy efficiency when the input traffic is high.
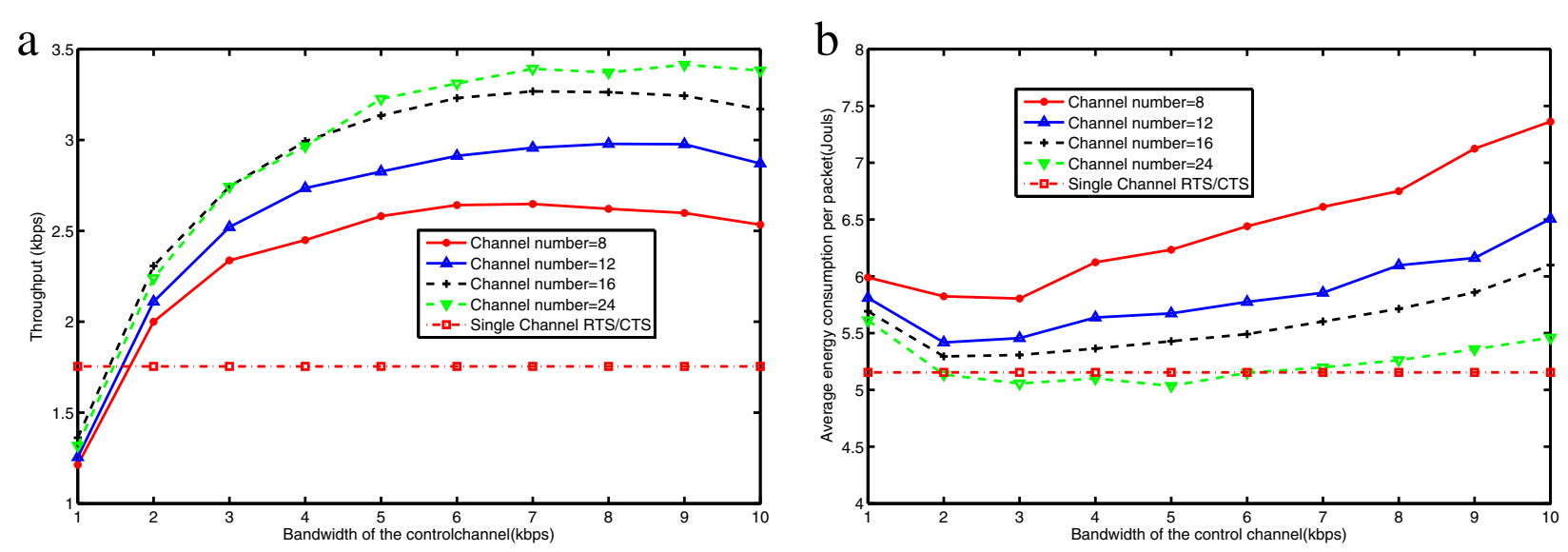

Figure 10 Comparison of RTS/CTS for single-channel and multi-channel. (a) Comparison of throughput. (b) Comparison of energy consumption. 


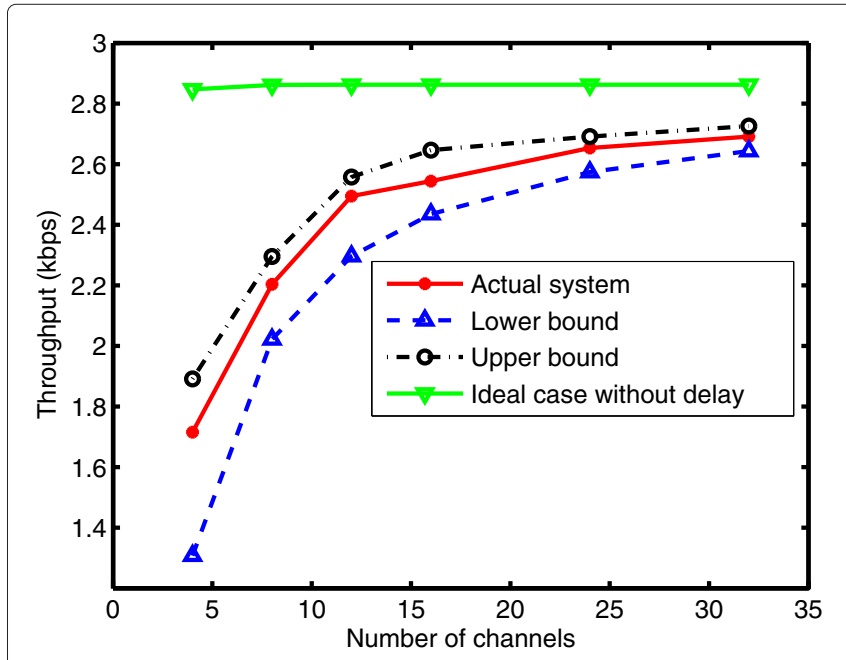

(a)

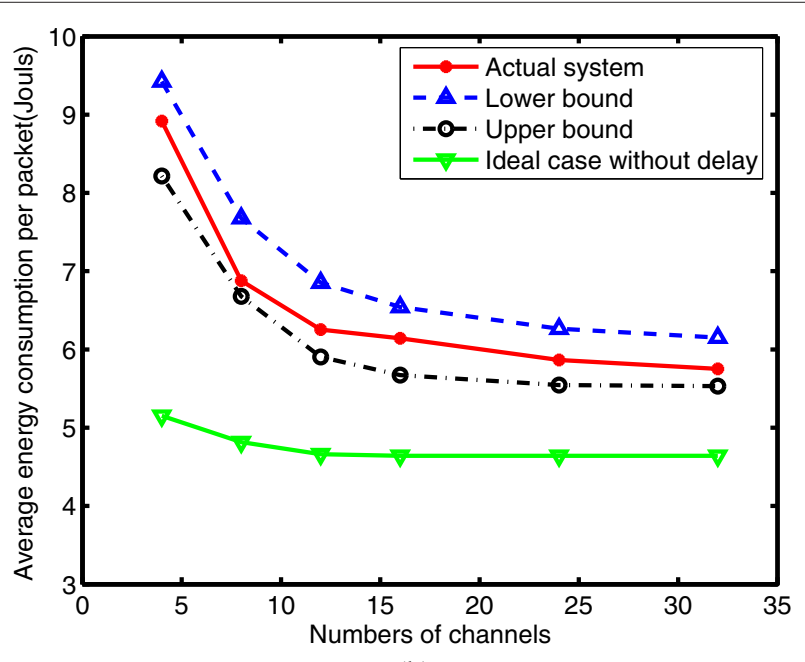

(b)

Figure 11 Comparison of bounds and actual system for "RTS/CTS" (with varying number of data channels). (a) Throughput; (b) average energy consumption.

\subsubsection{Effect of data packet length}

We increase the average length of data packets from 75 bytes to 750 bytes. The results are plotted in Figure 13. This figure shows that the packet size has significant impact on both random access and RTS/CTS approaches. Random protocols in both single-channel and multichannel networks show the same trend. when the packet length is low (less than 300 bytes), the network throughput has a limited increase, but decreases monotonically afterwards. This is because, for the random access protocol, the length of data packets has double effects. On the one hand, longer data packets may contribute to higher collision probability on the data channels, which might lead to the decrease of the throughput; On the other hand, potentially, with longer data packets, one successful data packet transmission will contribute more throughput than the case with shorter data packets. When the average length of data packets is short, the second factor dominates the first, therefore the network throughput increasing. But with the increase of data packet length, the first factor plays a major role and thus the network throughput will decrease. And as shown in Figure 13a, single-channel random access can achieve higher throughput than the multi-channel random access.

As shown in Figure 13, for single-channel RTS/CTS protocol, its throughput will first increase with the length of the data packet and then it will decrease slowly with it. This can be explained as follows. With the RTS/CTS exchange, the collisions of the data packets can be reduced, a longer data packet will contributes to a higher network throughput. However, Because of the long propagation delay of the underwater acoustic
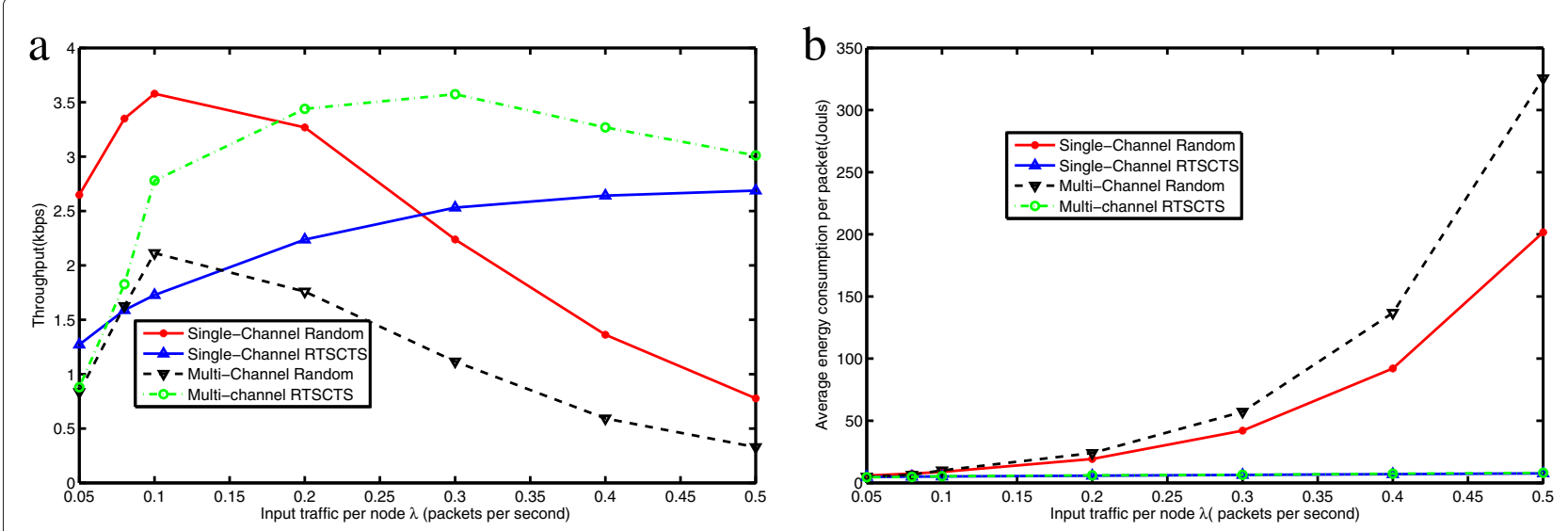

Figure 12 Performance comparison with varying input traffic $\lambda$. (a) Throughput. (b) Average energy consumption. 

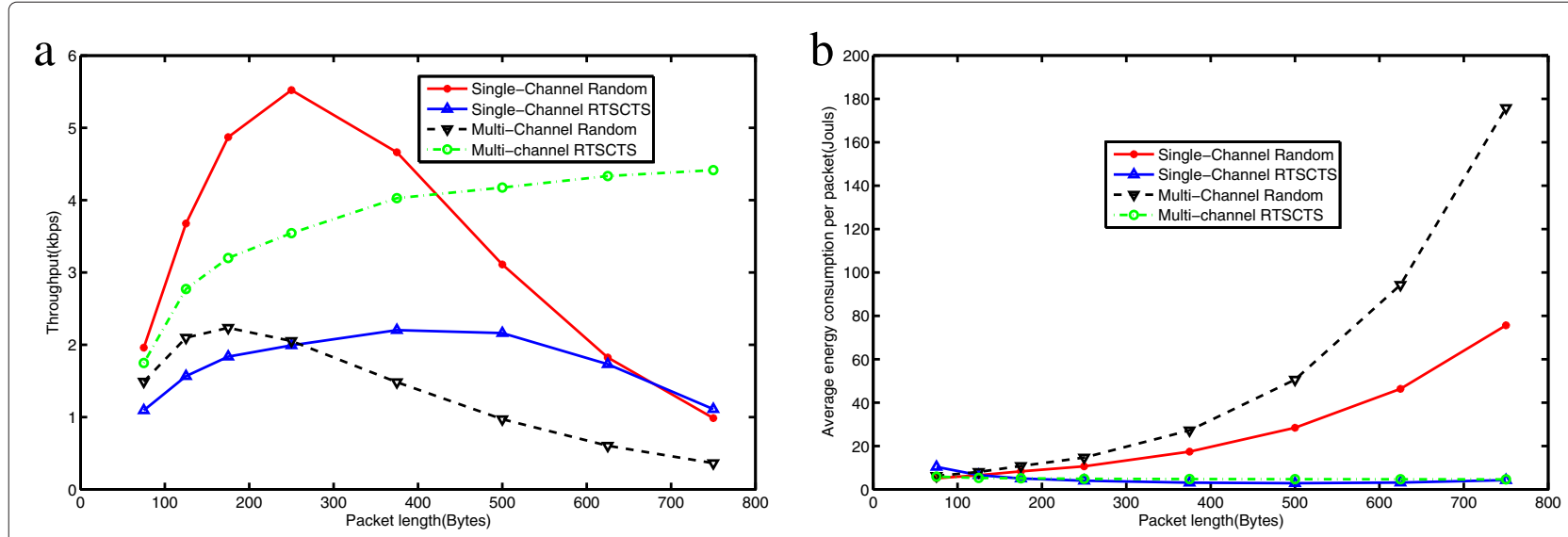

Figure 13 Performance comparison with varying data packet length. (a) Throughput. (b) Average energy consumption.

channel, the RTS/CTS exchange cannot totally eliminate the collisions of data packets, and a collision of a longer data packet will waste more system bandwidth, which will reduce the throughput. Thus, when the length of a data packet is too long(more than 500 bytes). While for the multi-channel RTS/CTS protocol, its throughput increases monotonically with packet length. This can be explained as follows. In multi-channel RTS/CTS protocol, its RTS/CTS handshaking process is conducted on a dedicated control channel, which will not be affected by the data packet. And the longer a data packet is, the more data will be transmitted in the data channels for each successful RTS/CTS exchange on the control channel. Thus a higher throughput will be achieved with longer data packet length.

From Figure 13b, we can also observe that the energy consumptions of the random access schemes in both single-channel and multi-channel protocols increase significantly with the data packet length. While for the RTS/CTS schemes, their average energy consumption decreases slowly with the increase of the average data packets length. And RTS/CTS schemes demonstrate much higher energy efficiency than the random schemes.

\subsubsection{Effect of propagation delays}

In this set of simulations, we change the average propagation delay from $0.1 \mathrm{~s}$ to $0.6 \mathrm{~s}$. This means the average distance between any nodes varies from $150 \mathrm{~m}$ to $900 \mathrm{~m}$. Figure 14 plots the results and demonstrates that the performance of the RTS/CTS protocols degrades a lot with the increase of the propagation delay. On the contrary, the performance of the random protocol is not affected by the propagation delay. This indicates "random access" is more robust for mobile networks with dynamic propagation delays and can provide a relatively stable performance to the upper layer. Thus, RTS/CTS schemes are not suitable for sparse networks with large propagation delay.

Compared with single-channel RTS/CTS scheme, multi-channel RTS/CTS is much more robust to the propagation delay. As shown in Figure 14a, when the average propagation delay increase from 0.2 second to $0.6 \mathrm{~s}$, the throughput of the single-channel RTS/CTS decreases almost $50 \%$ from 2.9 to 1.4 . While for multi-channel RTS/CST scheme, it only reduces from 2.9 to 2.4. Meanwhile, as shown in Figure 14b, Multi-channel RTS/CTS is also more energy efficient than the single-channel RTS/CTS protocol and the random access protocols when the propagation delay is long.

\subsection{Summary}

We summarize our simulation results as follows.

- (1) Random access has almost the same performance as or better performance than RTS/CTS under very low traffic and sparse deployment (which is consistent with the argument in [31]); while when the data traffic increases or the network gets denser, the channel is saturated quickly, resulting in low throughput degradation and high communication overhead; (2) In contrast, RTS/CTS has no significant advantages at low data rate with sparse network deployment, but provides more room for performance improvement in dense networks with high data rate; (3) The throughput of RTS/CTS is greatly affected by the transmission range. When the transmission range is large, RTS/CTS has a very low throughput. On the other hand, the transmission range has no effect on random access; (4) The packet size has significant impacts on the performance of both random access and RTS/CTS. In general, random access works 

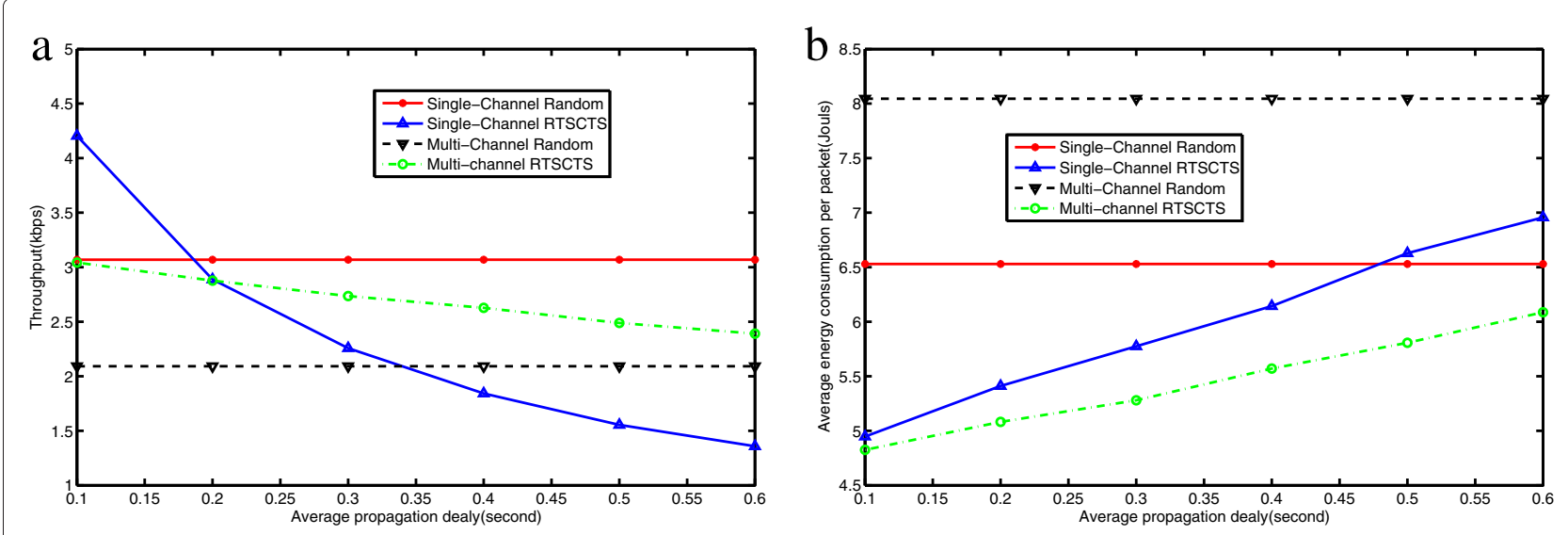

Figure 14 Performance comparison with varying propagation delays. (a) Throughput. (b) Average energy consumption.

better in networks with a small packet size; while RTS/CTS outperforms in large-packet-size networks;

- Multiple channels can potentially provide us one more degree of freedom to combat the long delay underwater acoustic environments. However, uncoordinated random channel access such as random access cannot fully exploit the advantages of the multi-channel network settings and it performs even worse than the single-channel counterparts. Only with careful design, can multi-channel MAC protocols improve the performance. For example, as shown in our simulations, compared with single-channel RTS/CTS, multi-channel with RTS/CTS can improve its throughput by almost $50 \%$.

\section{Related study}

In this section, we first summarize some recent MAC layer protocols for underwater acoustic networks. Next, we briefly review some related study on multi-channel MAC protocols for underwater acoustic networks and show their differences from our study.

Recently, efficient MAC protocols for long-delay underwater acoustic networks have received significant research attention. For example, in [21], to improve system performance, the authors minimize the duration of a hand-shaking process by taking advantages of receiver's tolerance to interference when two nodes are closer than the maximal transmission range. In [17], time is divided into slots with length of the maximal propagation delay. Transmissions are initiated at the beginning of slots. With strict synchronization, this scheme can greatly reduce the collision probability and is energy efficient. In [19], the authors propose that before data transmission, propagation delay measurement and channel reservation are made in advance to reduce the packet collision probability. In [22], the authors focus on the energy efficiency of the MAC protocol, data transmission is efficiently scheduled despite of the long propagation delay of acoustic channel in their proposed protocol. The authors of [23] study the Aloha-based protocols in underwater networks and propose two enhanced schemes, which take advantage of long propagation delays. A new MAC protocol, T-Lohi, appears in [39]. This protocol employs a novel tone-based reservation mechanism that exploits spacetime uncertainty and high latency to detect collisions and count contenders, achieving good throughput across all offered loads.

Multi-channel MAC protocols have long been investigated for terrestrial radio wireless networks [32-35]. In [35], multi-channel MAC protocols with Aloha like channel reservation have been modeled and analyzed. The authors of [34] compare multiple multi-channel MAC protocols through theoretical analysis and simulations. All these studies show that using multiple channels in parallel potentially can greatly improve the system performance. However, all of the above works are performed for radio communication where propagation delay is negligible. Their models and analysis processes can not be applied directly in the long-delay underwater environments.

Multi-channel schemes for underwater acoustic sensor networks have also aroused significant research interest recently. For example, in [40], a hierarchical multi-channel MAC protocols is proposed for clustered underwater networks where TDMA is used for the intra-cluster communication and CDMA is used for the inter-cluster communication. Strict synchronization among all nodes is needed in this scheme. In [41], the authors propose a random CDMA MAC protocols for underwater networks. In this scheme, one CDMA channel is dedicated as a control channel for control messages and every sender randomly chooses one of the CDMA channels for data transmission. This protocol can be treated as one instantiation of our generalized scheme of multi-channel with 
Aloha. The authors in this article focus their research on the optimal power control strategies in the physical layer. In [42], the authors utilize CDMA as the underlying multiple access technique. An RTS/CTS handshaking scheme is employed for every channel before actual data transmission. In this scheme, CDMA spreading codes are distributed before hand by some predefined algorithm and every node is assumed to get a unique spreading code among its one-hop neighbors.

Summarizing all these research efforts on the MAC protocols for underwater acoustic networks, none of them have theoretically modeled, analyzed and compare the most basic schemes, namely random access and handshaking, for the long-delay harsh underwater environment, which is the focus of our article.

\section{Conclusions}

In this article, we have formally studied the random access and handshaking (i.e., RTS/CTS) techniques in underwater acoustic networks. We modeled and analyzed these two approaches in both single-channel and multi-channel network scenarios, and then conducted extensive simulations to investigate their performance in various network conditions. Based on our results, we conclude that the simple random access approach is preferred in sparse networks with very low data traffic; while RTS/CTS has better performance when the network gets denser and the data rate becomes higher. Moreover, the performance of RTS/CTS degrades with the increase of the propagation delay; while the propagation delay has almost no impacts on the random access. Multiple channels potentially can improve the system's performance. However, uncoordinated random channel access such as random access cannot fully exploit the advantages of the multichannel network settings. Only with careful design, can multi-channel MAC protocols improve the system performance.

Recalling the three questions raised in the introduction, we are now in a good shape to answer them: (1) Random access is not an absolute winner; (2) RTS/CTS can achieve better performance in many network conditions (as summarized earlier); (3) Multiple channel technologies can provide higher performance with careful protocol design. Our study indicates that it is possible to design an adaptive MAC protocol, adopting random access and RTS/CTS dynamically for different networks conditions.

\subsection{Future study}

We would like to pursue our study in following directions: (1) Devise an adaptive MAC protocol to accommodate the complex conditions in underwater acoustic networks. (2) In this study, we ignore the effects of low rate underwater acoustic modems with long preambles on the MAC protocol. Theoretical analysis on this practical issue is very interesting and can provide us more guidelines on the MAC protocol design for practical underwater networks. (3) The multi-channel technology has double effects on the overall packet latency. On one hand, the introduction of multiple channels will increase the packet transmission time. On the other hand, multiple channels potentially can decrease the collision probability in the network, which will decrease the average packet latency. Analysis on the packet latency in the multi-channel underwater network is another interesting research topic.

\section{Endnotes}

a In the market, "AquaNetwork" modems from DSPCOMM can provide multiple channels to the upper layer [43].

b In underwater acoustic communication, data receiving and processing consume much less energy than data transmitting [44].

c For some low acoustic modems with long preambles, the collisions and the transmission delay of the control packets are not negligible anymore. which will degrade the performance of MAC protocols.

d In this article, we only consider the one-hop network scenario. For multi-hop networks, one more control message is needed on the sender side to inform its own neighbors, which are not the receiver's neighbors, of this communication event as in [33].

e For multi-channel case, the collisions for the RTS/CTS messages on the control channel is not negligible anymore. This is because the transmission time for RTS/CTS message is much longer than that for the single-channel case considering the bandwidth of the control channel dedicated for the RTS/CTS like control message is much smaller than the total bandwidth in the network.

\section{Appendix 1}

\section{Calculation of the lower bound for multi-channel with RTS/CTS}

Here, the system status is set as the number of the available data channels in a slot and we use $x_{i}$ to denote the system status in slot $i$. The next status $x_{i+1}$ will be shown to be only related to the current status $x_{i}$.

The one-step transition probability from state $x_{i}=y_{0}$ to $x_{i+1}=y_{1}$ can be denoted as

$$
P\left(x_{i+1}=y_{1} \mid x_{i}=y_{0}\right)=P\left(x_{i+1}-x_{i}=y_{1}-y_{0} \mid x_{i}=y_{0}\right) \text {. }
$$

Set $B$ to be the number of channels that have been used by the newly arrived packets in slot $i, A$ to be the number 
of channels that have been released (because of the end of one packets) in slot $i$. Then, we can get that

$$
x_{i+1}-x_{i}=A-B .
$$

If $x_{i}=y_{0}$ and there are $s$ incoming packets during the $i$ th slot, the probability that these packets occupy exactly $k$ channels can be written as,

$$
P\left(B=k \mid s, x_{i}=y_{0}\right)=\sum_{j=0}^{k}(-1)^{(k-j)}\left(\begin{array}{l}
k \\
j
\end{array}\right)\left(\frac{j}{y_{0}}\right)^{s} ; k \leq s .
$$

Then, the distribution of $\mathrm{B}$ when $x_{i}=y_{0}$ can be calculated as follows,

$$
\begin{aligned}
& P\left(B=k \mid x_{i}=y_{0}\right) \\
& =\sum_{s \geq k} P(s) P\left(B=k \mid s, x_{i}=y_{0}\right) \\
& =\sum_{s \geq k}\left[\frac{\left(2 \lambda_{e} \tau\right)^{s}}{s !} e^{-2 \lambda_{e} \tau} \sum_{j=0}^{k}(-1)^{(k-j)}\left(\begin{array}{l}
k \\
j
\end{array}\right)\left(\frac{j}{y_{0}}\right)^{s}\right] \\
& =\sum_{s \geq k} \sum_{j=0}^{k}\left[(-1)^{(k-j)} \frac{\left(2 \lambda_{e} \tau\right)^{s}}{s !} e^{-2 \lambda_{e} \tau}\left(\begin{array}{l}
+k \\
j
\end{array}\right)\left(\frac{j}{y_{0}}\right)^{s}\right] \\
& =e^{-2 \lambda_{e} \tau} \sum_{j=0}^{k}(-1)^{k-j}\left(\begin{array}{l}
k \\
j
\end{array}\right)\left(e^{-2 \lambda_{e} \tau} \frac{j}{y_{0}}\right. \\
& \left.\quad-\sum_{s<k} \frac{\left(2 \lambda_{e} \tau\right)^{s}}{s !} e^{-2 \lambda_{e} \tau}\left(\frac{j}{y_{0}}\right)^{s}\right)
\end{aligned}
$$

Set $\mu=\frac{1}{T_{d}}$, where $T_{d}$ is the average transmission time for one data packet. When the packet length is subject to an independent exponential distribution, the distribution of $A$ then can be calculated as

$$
\begin{gathered}
P\left(A=k \mid x_{i}=y_{0}\right)=\left(m-y_{0} k\right)\left(1-\mu e^{-2 \mu \tau}\right)^{k} \\
\times\left(\mu e^{-2 \mu \tau}\right)^{\left(m-y_{0}-k\right)} \quad 0 \leq k \leq m-y_{0}
\end{gathered}
$$

And thus, we can get that

$$
\begin{gathered}
P\left(x_{i+1}=y_{1} \mid x_{i}=y_{0}\right)=P\left(x_{i+1}-x_{i}=y_{1}-y_{0} \mid x_{i}=y_{0}\right) \\
=\sum_{k=0}^{y_{0}} P\left(B=k \mid x_{i}=y_{0}\right) P\left(A=k+\left(y_{1}-y_{0}\right) \mid x_{i}=y_{0}\right) \\
=\sum_{k=0}^{y_{0}} e^{\left(-2 \lambda_{e} \tau\right)}\left\{\sum _ { j = 0 } ^ { k } \left[( - 1 ) ^ { k - j } ( \begin{array} { l } 
{ k } \\
{ j }
\end{array} ) \left(e^{-2 \lambda_{e} \tau \frac{j}{y_{0}}}\right.\right.\right. \\
\left.\left.\left.\quad-\sum_{s<k} \frac{\left(2 \lambda_{e} \tau\right)^{s}}{s !}\left(\frac{j}{y_{0}}\right)^{s} e^{\left(-2 \lambda_{e} \tau\right)}\right)\right]\right\}\left(\begin{array}{l}
m-y_{0} \\
k+\left(y_{1}-y_{0}\right)
\end{array}\right) \\
\quad \times\left(1-\mu e^{-2 \mu \tau}\right)^{k+\left(y_{1}-y_{0}\right)} \times\left(\mu e^{-2 \mu \tau}\right)^{m-k-y_{1}} ; \\
m-y_{0} \geq k+\left(y_{1}-y_{0}\right) .
\end{gathered}
$$

Equation (24) gives us the one-step transition probability of lower bound system from state $y_{0}$ to $y_{1}$. We can clearly see that it is only related to the current system status, Now, we can get the one step transition matrix $P_{T}$, based on which we can easily calculate the system stationary probability $P_{\text {st }}$ and the collision probability $P_{\text {col }}$. In the same way, we can prove that the upper bound system can also be modeled by a discrete Markov chain and we can also calculate its stationary probability and average collision probability.

\section{Competing interests}

The authors declare that they have no competing interests.

\section{Author details}

${ }^{1}$ Computer Science \& Engineering Department, University of Connecticut, Storrs, CT, USA. ${ }^{2}$ Acoustic Division Naval Research Lab, Washington DC, USA.

Received: 12 July 2012 Accepted: 17 January 2013 Published: 02 April 2013

\section{References}

1. IF Akyildiz, D Pompili, T Melodia, Underwater acoustic sensor networks: research challenges. Ad Hoc Netw. J. (Elsevier). 3(3), 257-281 (2005)

2. J Heidemann, W Ye, J Wills, A Syed, Y Li, in Proceedings of IEEE Wireless Communications and Networking Conference, vol. 1. Research Challenges and Applications for Underwater Sensor Networking (Las Vegas, Nevada, USA, 2006), pp. 228-235

3. J-H Cui, J Kong, M Gerla, S Zhou, Challenges: building scalable mobile underwater wireless sensor networks for aquatic applications. IEEE Netw. Special Issue on Wireless Sensor Networking. 20(3), 12-18 (2006)

4. J Partan, J Kurose, BN Levine, in Proceedings of ACM WUWNet'06, vol. 1. A survey of practical issues in underwater networks (Los Angeles, CA, USA, 2006), pp. 17-24

5. L Liu, S Zhou, J-H Cui, in Wiley Wireless Communications and Mobile Computing, Special Issue on Underwater Sensor Networks, vol. 8(8). Prospects and problems of wireless communications for underwater sensor networks, (2008), pp. 977-994

6. D Pompil, T Melodia, IF Akyildiz, in Proceedings of the 12st Annual International Conference on Mobile Computing and Networking (Mobicom) vol. 1. Routing algorithms for delay-insensitive and delay-sensitive applications in underwater sensor networks (Los Angeles, CA, USA, 2006), pp. 298-309

7. P Xie, L Lao, J-H Cui, in Proceedings of IFIP Networking, vol. 1. VBF: vector-based forwarding protocol for underwater sensor networks (Coimbra, Portugal, 2006), pp. 1216-1221

8. D Pompili, T Melodia, in Proceedings of the 2nd ACM International Workshop on Performance Evaluation of Wireless Ad Hoc, Sensor, and Ubiquitous Network vol. 1. Three-dimensional routing in underwater acoustic sensor networks (New York, NY, USA, 2005), pp. 214-221

9. V Rodoplu, MK Park, in Proceedings of the MTS/IEEE OCEANS, vol. 2. An energy-efficient MAC protocol for underwater wireless acoustic networks (Washington, DC, USA, 2005), pp. 1198-1203

10. P Xie, J-H Cui, in Proceedings of the MTS/IEEE OCEANS, vol. 1. Exploring random access and handshaking techniques in large-scale underwater wireless acoustic sensor networks (Boston, MA, USA, 2006), pp. 1-6

11. Peleato $B$, Stojanovic $M$, in Proceedings of the 1st ACM international workshop on Underwater networks, vol. 1. A MAC protocol for ad-hoc underwater acoustic sensor networks (New York, NY, USA, 2006), pp. 113-115

12. Syed A A, Heidemann J, in Proceedings of the 25th IEEE International Conference on Computer Communications (INFOCOM'06), vol. 1. Time synchronization for high latency acoustic networks (Barcelona, Spain, 2006), pp. 1-12

13. R Jurdak, CV Lopes, P Baldi, Battery lifetime estimation and optimization for underwater sensor networks. IEEE Sensor Netw. Operat. 1, 397-420 (2004) 
14. P Xie, Z Zhou, Z Peng, J-H Cui, SDRT: a reliable data transfer protocol for underwater acoustic sensor networks. Ad Hoc Networks 8(7), 708-722 (2010)

15. Z Zhou, Z Peng, J-H Cui, Z Shi, Energy efficient multi-path communication for time-critical applications in underwater sensor networks. IEEE/ACM Trans. Network. 21, 28-41 (2011)

16. Z Zhou, H Mo, Y Zhu, J-H Cui, J Huang, in IEEE ICC, vol. 1. A new fountain code based reliable data transfer protocol for long-delay underwater acoustic networks (2012), pp. 1-6

17. M Molins, M Stojanovic, in Proceedings of the IEEE Oceans Conference, vol. 1. Slotted FAMA: a MAC protocol for underwater acoustic networks (Singapore, 2006), pp. 1-7

18. P Xie, J-H Cui, in Proceedings of MTS/IEEE OCEANS, vol. 1. Exploring random access and handshaking techniques in large-scale underwater wireless acoustic sensor networks (2006) (Boston, MA, USA, 2006). pp. 1-6

19. P Xie, J-H Cui, in Proceedings of International Conference on Wireless Algorithms, Systems, and Applications (WASA'07), vol. 1. R-MAC: an energy-efficient MAC protocol for underwater sensor networks (Chicago, Illinois, USA, 2007), pp. 187-198

20. KB Kredo, P Mohapatra, in Proceedings of ACM WUWNet'07, vol. 1. A hybrid medium access control protocol for underwater wireless networks (New York, NY, USA, 2007), pp. 33-40

21. B Peleato, M Stojanovic, Distance aware collision avoidance protocol for ad-hoc underwater acoustic sensor networks. IEEE Commun. Lett. 11(12), 1025-1027 (2007)

22. MK Park, V Rodoplu, UWAN-MAC: an energy-efficient mac protocol for underwater acoustic wireless sensor networks. IEEE J. Ocean. Eng. 32(3), 710-720 (2007)

23. N Chirdchoo, W-S Soh, KC Chua, in Proceedings of INFOCOM'07, Mini-Symposium, vol. 1. Aloha-based MAC protocols with collision avoidance for underwater acoustic networks (Anchorage, AK, 2007), pp. 2271-2275

24. A Syed, W Ye, J Heidemann, T-Lohi: a new class of MAC protocol for underwater acoustic sensor networks vol. 1, (Phoenix, AZ, USA, 2008), pp. 231-235

25. N Abramson, The ALOHA System-Another Alternative for Computer Communications, vol. 37. (AFIP Press, New York, NY, USA, 1970)

26. LG Robert, in ACMSOGCOMM Comput. Communication review, vol. 39. ALOHA packet system with and without slots and capture (New York, NY, USA, 1975), pp. 28-42

27. P Karn, in Proceedings of the ARRL/CRRL Amateur Radio 9th Computer Networking Conference, vol. 39. MACA-A new channel access method for packets radio (London, Ontario, Canada, 1990), pp. 134-140

28. $\vee$ Bhargavan, A Demers, S Shenker, L Zhang, MACAW-A medium access protocol for wireless LANs, ACM SIGCOMM, vol. 24, (New York, NY, USA 1994), pp. 212-225

29. JG Proakis, EM Sozer, JA Rice, M Stojanovic, Shallow water acoustic networks. IEEE Commun. Mag. 39(11), 114-119 (2001)

30. IF Akyildiz, D Pompili, T Melodia, Challenges for efficient communication in underwater acoustic sensor networks. ACM Sigbed Rev. 1(1) (2004)

31. V Rodoplu, MK Park, in IEEE Oceans Conference, vol. 2. An energy-efficient MAC protocol for underwater ireless acoustic networks (Washington DC, USA, 2005), pp. 1198-1203

32. A Tzamaloukas, JJ Garcia-Luna-Aceves, in Proceedings of INFOCOM, vol. 1. A receiver-initiated collision-avoidance protocol for multi-channel networks (Anchorage, AK, 2001), pp. 189-198

33. J So, N Vaidya, in Proceedings of ACM MobiHoc, vol. 1. Multi-channel mac for ad hoc networks: Handling multi-channel hidden terminals using a single transceiver (Tokyo, Japan, 2004), pp. 222-233

34. J Mo, H-S Wilson, J Walrand, Comparison of multichannel mac protocols. IEEE Trans. Mob. Comput. 7(1), 50-65 (2008)

35. YS Han, J Deng, ZJ Haas, Analyzing multi-channel medium access control schemes with ALOHA reservation. IEEE Trans. Wirel. Commun. 5(8), 2143-2152 (2006)

36. MA Bhatti, Practical Optimization Methods with Mathematical Applications. (Springer, New York, 2000)

37. JL Doob, Stochastic Processes. (John Wiley and Sons, New York, 1953)

38. FA Tobagi, L Kleinrock, Packet switching in radio channels: Part III-polling and (dynamic) split-channel reservation multiple access. IEEE Trans. Commun. 24(8), 832-845 (1976)
39. AA Syed, W Ye, J Heidemann, in Proceedings of Infocom, vol. 1. T-Lohi: a new class of MAC protocols for underwater acoustic sensor networks (Phoenix, AZ, USA, 2008), pp. 231-235

40. F Salva-Garau, M Stojanovic, in Proceedings of the IEEE Oceans Conference, vol. 1. Multi-cluster protocol for ad hoc mobile underwater acoustic networks (San Diego, CA, USA, 2003), pp. 91-98

41. D Pompili, T Melodia, IF Akyildiz, in Proceedings of Mediterranean Ad HoC Networking Workshop, vol. 1. A distributed cdma medium access control for underwater acoustic sensor networks (Corfu, Greece, 2007), pp. 64-72

42. H-XW Tan, KG Seah, in Proceedings of the 32th IEEE Conference on Local Computer Networks, vol. 1. Distributed cdma-based mac protocol for underwater sensor networks (Dublin, 2007), pp. 26-36

43. Aquanetwork: underwater wireless modem with networking capability. http://www.dspcomm.com/products_aquanetwork.html

44. L Freitag, M Grund, S singh, J Partan, P Koski, K Ball, in Proceedings of MTS/IEEE OCEANS, vol. 2. The WHIO micro-modem: an acoustic communications and navigation system for multiple platforms (Washington, DC, USA, 2005), pp. 1086-1092

doi:10.1186/1687-1499-2013-95

Cite this article as: Zhou et al:: Exploring random access and handshaking techniques in underwater wireless acoustic networks. EURASIP Journal on Wireless Communications and Networking 2013 2013:95.

\section{Submit your manuscript to a SpringerOpen ${ }^{\odot}$ journal and benefit from:}

- Convenient online submission

Rigorous peer review

- Immediate publication on acceptance

- Open access: articles freely available online

- High visibility within the field

- Retaining the copyright to your article

Submit your next manuscript at springeropen.com 